the cause which led to the use of the forceps in 29 of my cases, and even a very considerable degree of disproportion may be thus overcome.

It should not be concluded, because a woman has some pelvic deformity, and because her medical attendants in former labours delivered her by craniotomy or cephalotripsy, that we should follow this example. I have applied the forceps in several cases in which craniotomy had been previously resorted to on account of some disproportion. It should never be forgotten that a living child may pass through a pelvis scarcely measuring three inches in its widest diameter, and that, therefore, even a considerable degree of pelvic deformity may admit of the delivery of a living child by the long forceps.

I have also used this forceps in cases of breech-presentation, in which the head was prevented from passing the brim by malposition, and also in head-presentations delayed in the same way. Long delay in labour from a rigid unyielding condition of the cervix uteri led to this operation in several of the cases reported in the foregoing table. The other cases in which I have resorted to the long forceps were chiefly instances of labour complicated by convulsions, by prolapse of the cord, by accidental hæmorrhage before the head had entered the cavity, by exhaustion, by threatened rupture; and in one instance I employed this instrument to effect delivery in a case of rupture of the uterus.

In such cases, where the symptoms are urgent, I have not considered it necessary to wait for the full dilatation of the os, and have never hesitated to employ this instrument, when circumstances rendered it necessary as soon as the os was dilatable, or could be sufficiently exposed.

Still, nothing but the most absolute necessity should ever induce us to deviate from the time-honoured rule that the os should be fully dilated before the forceps are resorted to.

Another rule of great value, though now very frequently disregarded, is that, in ordinary cases of difficult labour from inertia, the forceps should not be resorted to until a trial has been given to other means, such as friction over the uterus, change of position, and stimulating enemata of warm water and salt.

These having failed, and the application of the forceps having been first sanctioned by a consultation, and the bladder being emptied by the catheter, the position of the head should be ascertained immediately before the operation, and a dose of ergot should be administered. The patient is then generally placed under the influence of chloroform, and if the short forceps are to be used she is drawn to the edge of the bed in the usual left lateral semiprone position. The operator should sit facing the perinæum, introducing the index and second fingers of the right hand within the vagina behind the symphysis pubis, and feel for the ear of the child. This is deemed unnecessary by some writers; but when the short forceps is used it should be practised, inasmuch as the instrument, fitting better over the sides of the head and face, exerts a more equal pressure, and is less liable to slip than when placed in any other position. The instrument being first warmed and oiled, the upper or pubic blade is to be taken in the operator's left hand as light as a writing pen, and gently insinuated in the axis of the pelvis, by a sligh wavy motion, between the two forefingers of his right hand and the child's head until the lock rests on the perinæum. In the same manner, using no force whatever, the second or sacral blade is to be introduced, the operator reversing the position of his hands. As soon as the locking is completed, the head is to be very gently drawn downwards and backwards, and then downwards and forwards until the vertex begins to emerge from the vulva, when the blades should be withdrawn, and the head helped out by pressure with the hand on the perinæum.

With regard to the application of my long forceps, I have only to say that it is most easily accomplished when the patient is placed on her back and draw down to the foot of the bed; but, when she objects to this, it may be readily accomplished by drawing her hips well over the edge of the bed, and following the same course as that directed for the application of the short forceps, with the exception that it is not so essential to be particular about the position of the child's head, as the instrument must be applied in the largest or transverse diameter of the pelvis, one blade being in each ilium; and hence, as I pointed out in a work to which I have before referred, it is a matter of comparatively little importance whether they are applied antero-posteriorly or transversely over the child's head.

The short forceps now exhibited is, I believe, about the safest instrument of the kind that can be employed, and the long double curved one I hold in my hand is, perhaps, the most powerful. But I must add that an instrument so powerful for evil as well as for good, as any long double curved forceps must be, is not to be rashly resorted to. And when we remember that Smellie, who first introduced the long orceps into this country, was so fearful of the possible abuse of its powers as to abstain from recommending its use, or even from exhibiting it to his class, we should at least exercise no ordinary discretion in our freer use of what an old writer terms " this noble instrument".

\section{REPORT OF A CASE OF PARACENTESIS THORACIS.*}

By G. H. PHILIPSON, M.A., M.D.Cantab., F.R.C.P.Lond., Physician to the Newcastle-upon-Tyne Infirmary, etc.

PATRICK O'NeIL, aged 22, single, labourer, was admitted into the Newcastle-upon-Tyne Infirmary, under my care, March 5th, I874. He stated that he had been unable to follow his employment for two weeks, but that he had not been well for three months, having suffered from cough; and that, at the commencement of his illness, he had experienced a severe sharp pain in the left side of his chest, which was made worse by breathing.

At the time of his admission, he complained greatly of difficulty in breathing, of thirst, and of general weakness. On inspection of the chest, it was seen that the left side was stationary during forced respiration, and appeared larger than the right; and that the intercostal spaces were widened, the depressions being effaced. On percussion, the left side was found to be wholly dull, in front from the clavicle to the lower boundary of the chest-wall, and behind as high as the spine of the scapula. Respiration, vocal resonance and fremitus, and tussive resonance, were absent. On the right side, the respiratory sounds were puerile in character, aecompanied with sonorous rhonchus; while the voice-resonance and fremitus were very distinct. The right side of the chest at the nipple-line measured $16 \frac{7}{8}$ inches, and the left side $17 \frac{1}{8}$ inches; the left being a quarter of an inch larger than the right. The heart's impulse was visible and perceptible two and a half inches to the right of the sternum. The sounds were distinct and without murmur. The pulse was I2O, hard and small ; the temperature was IO3 deg. Fahr. He was much emaciated, the biceps readily starting into ridges upon being percussed. He was of the lymphatic temperament, having dark-brown hair, long eyelashes, dark grey eyes, and thick upper lip. He was ordered quinine, and to have the chest well rubbed every night and morning with the liniment of turpentine and acetic acid.

March IIth. The urgency of the symptoms had so increased as to denote serious interference with the functions of life, and it was determined to afford relief by paracentesis. The operation was performed by Mr. George Rowell, junior house-surgeon, with the aspirator, when five-and-a-half pints of a yellowish-coloured clear liquid was withdrawn, which became semi-solid after a short interval. The liquid continued to flow for one hour and a quarter, and cluring the time the patient was frequently required to inspire deeply. During the passage of the liquid, the heart was observed gradually to resume its normal position, and finally was seen beating in the fifth intercostal space on the left side, about one inch below, and half an inch within, the left nipple. After the operation, the patient appeared much relieved, and voluntarily expressed his comfort. Respiration was then clearly heard in the left infraclavicular region, as also the vocal resonance. He was ordered ten grains of the compound powder of ipecacuanha at bedtime.

March I 8th. For the last three days, he had been able to be out of bed, and to walk about the ward. He expressed himself as able to breathe with ease, but still complained of thirst and weakness. His pulse was 120, and temperature I03 deg. Fahr. Vocal fremitus was distinctly perceptible over the left back as low as the eighth rib. Respiration and vocal resonance were also distinctly heard. The percussion-note was clear from the eighth rib upwards. The respiration on the right side was much less puerile, and unaccompanied with rhonchus. The measurement of the right side of the chest at the nipple-line was $\mathbf{I} 7$ inches, and the left $\mathbf{I} 7$ inches. He was ordered cod-liver oil in combination with the syrup of the iodicle of iron, three ounces of port wine, and the full meat-diet.

March 25th. He appeared stronger, and complained less of thirst. His pulse was IIO, and temperature IO2 Fahr. Respiration was clearly heard at the left base; also vocal resonance.

April 8th. His weight was 8 st. $3 \frac{1}{2} \mathrm{lb}$. Pulse 100 , and temperature IOO deg. Fahr.

April 22nd. His weight was 8 st. 5 1/2 lb. Pulse roo, and temperature 98 deg. Falhr. He was able to be out in the garden every fine day, and was taking his food with relish. It was noted that his shoulders were equal in height, and that there was no curvature of the spine.

REMARKS. - This case has been recounted with the riew of directing attention to the value of the operation of paracentesis in cases of 
extensive pleuritic effusion, not only in preventing a fatal result from impending suffocation, but also in expediting the restoration of the patient ; the simplicity, painlessness, and freedom from danger of the operation by the aspirator being well exemplified. It also illustrates the necessity of not delaying the operation until the lung has become totally unfitted for free expansion, and displaced organs have become permanently fixed by pleuritic adhesions. The interest of the case is further increased by the considerable and long continued elevation of the temperature, and the prolonged quickness of the pulse. In the chart, extended over a period of five weeks, a range of temperature is indicated between I03.6 and 98 deg. Fahr., and a pulse between I 20 and roo. When the temperature and pulse are reviewed together, and with the emaciation and temperament superadded, the deposition of tubercle is indicated, and the fear that the pleurisy was diathetic, and not purely inflammatory, predicated.

\section{A CASE OF EPITHELIOMA OF THE BODY OF THE UTERUS TREATED BY DILATATION, THE CURETTE, AND LOCAL APPLICA- TION OF TINCTURE OF IODINE.}

By THOMAS CHAMBERS, M.R.C.P., F.R.C.S.Edin., Physician to the Chelsea Hospital for Women, etc.

MRs. T., aged 56 , widow of a naval officer, had been married twenty years, but had never been pregnant. Menstruation was regular up to her fiftieth year, when it ceased, without inconvenience or irregularity of any kind. For four years she continued to enjoy good health. About this time, she became subject to sudden and frequently repeated attacks of uterine hæmorrhage, occurring at intervals of six or eight weeks. Sometimes the discharge continued two or three days; at others, six or eight days, or even longer. During the two years that this discharge had continued, she had consulted different medical men, who differed considerably as to the cause of the hæmorrhage.

I saw this lady for the first time on January Ioth, 1873. She appeared to be in pretty good general health, though she thought herself thinner than she was three months before. For some time she had had a constant feeling of depression, without being able to account for it - a condition most unusual to her. She had no pain of any kind, excepting an occasional shoot through the groins and along the lower part of the abdomen. These pains were not frequent; and when they did occur, they quickly passed away. Her only trouble was the frequent vaginal hæmorrhages. Pulse 98; temperature 99; no cough, perspirations, or shiverings.

On a careful examination of the abdomen, nothing could be seen or felt save a small round body immediately behind the pubic arch, which could be readily pressed down into the pelvis. There was no enlargement of the glands of the groins. The vagina was perfectly healthy as far as the touch could be a guide. The os and cervix uteri were also healthy and natural to the touch. The body of the uterus was, however, a little larger than it ought to be at the patient's period of life. By placing the right hand on the round body above the pubes, and the left index finger against the os and cervix uteri, the uterus could be accurately measured, and freely moved in any direction without pain or inconvenience, there being no thickening or deposit of any kind in the pelvis. The sound passed with ease three inches and three-quarters. When the fundus was touched by the end of the sound, a sharp shooting pain was experienced, such as she had felt before through the groins. The case being regarded as one of polypus, the sound was gently moved about the cavity without detecting anything beyond a general unevenness of surface. On withdrawing the instrument, the hæmorrhage was so profuse as to necessitate the plugging of the vagina with cotton-wool saturated in tincture of perchloride of iron. This free hæmorrhage, associated with the nodular surface of the uterine cavity revealed by the sound, the occasional shooting pains through the groins and pelvis, and the faintish smell imparted to the finger while examining the uterus, these facts, when in association, pointed to the case as one of probable malignant disease of the body of the uterus, rather than polypus. The loss sustained on this occasion was considerable, and the patient did not recover from its effects for ten or twelve days. As her means were limited, she was admitted into a private room in the Chelsea Hospital for Women on January 22nd, I873.

January 24 th. A tangle-tent was introduced, as large as the os and cervix would admit.

January 25 th. On withdrawing the tent, a free flow of blood followed, intermixed with small granular bodies. These, under the microscope, proved to be little masses of cells of a peculiar shape, being for the most part bottle-shaped. These facts supported the opinion as to the malignant nature of the disease. The cavity was washed out with perchloride of iron, and three fresh tents introduced.

January 26th. The cervix being now fully dilated, she was put under chloroform. The anterior lip being fixed with a vulsellum, the left index finger was passed into the uterine cavity, which was lined by nodular growths differing considerably in size, there being no healthy surface recognisable. About half way up the uterus, there could be felt what appeared to be a number of these nodules strung together, forming a band across the cavity from side to side, divided to some extent into an upper and a lower part. My colleague, Dr. Aveling, examined the case with me.

The nodules or vegetations were soft and friable, bleeding freely at the slightest touch. We were both of opinion that this was a well marked case of malignant disease of the body, chiefly submucous, as far as could be made out. Holding the uterus fixed by the vulsellum, I scraped out the cavity freely with Sims's curette until every nodule had been removed, leaving a smooth uniform surface throughout the cavity. When this had been accomplished, all hæmorrhage ceased. The cavity was well wiped out with dry cotton-wool, and then swabbed with the compound tincture of iodine; a suppository was put into the bowel, and the patient removed to bed. She felt faint and depressed for a few hours after the operation, but this soon passed off. For ten days, there was a free discharge of dark grumous fluid from the vagina, which was washed out twice daily with carbolic acid lotion. The patient was well nourished, and had a moderate amount of wine and stout daily. A mixture of quinine, iron, and Fowler's solution, was taken thrice daily; a chloral draught at bedtime when necessary.

February I 9 th. A month had passed since the operation, without any return of the hrmorrhage; neither had there been any vaginal discharge for the last ten or twelve days. The shooting pains in the groins had not been noticed during the last three weeks. The vagina, os, and cervix uteri, were all healthy, and in much the same state as before the operation. The sound passed three inches without detecting any irregularity of surface, such as was noticed when first examined; and, notwithstanding the freedom with which the instrument was moved about in the cavity, there was no hæmorrhage. The general health had improved; she felt herself again, and was anxious to go to the country. She was discharged at her own request. External palpation showed that the round body, which was noticed behind the pubes when first examined, had to a great extent disappeared.

On a careful review of the whole case, the age of the patient, the sudden appearance of hæmorrhage after menstruation had ceased for four years, the alarming hæmorrhage which followed the examination of the uterus by the sound, the microscopical appearances of the cellgrowths, and the general physical signs observed by Dr. Aveling and myself, cannot, I think, admit of any doubt as to the case being one of malignant disease of the body of the uterus. The results of treatment have so far (July 1873) been satisfactory; but there is every reason to fear that time only is necessary for the reappearance of the disease. Should this case again come under my notice under circumstances so favourable-viz., freedom from complication with surrounding parts, the cervix uteri, groin-glands, etc. - I must confess that the question of gastrotomy, with a view to the removal of the entire organ down to the cervix, ought not to be overlooked.

\section{ON THE CONSTRICTOR ACTION OF THE INTER- COSTAL MUSCLES.}

BY ARTHUR RANSOME, M.D., M.A.,

Late Examiner in Anatomy, Physiology, and Pharmacology to Cambridge University (1868-1869).

AMONGST the various actions which have been attributed by different observers to the two layers of intercostal muscular fibres, no note appears to have been taken of a function which may, I think, be fairly assigned to them, namely, the indrawing or constriction of the walls of the thorax. It is probable that the supposition of the rigidity of the bony levers (the ribs) has in some measure prevented the recognition of this action; and the fact that constriction of the chest-wall takes place only during forced breathing, would prevent it from being remarked in experiments upc $n$ the ordinary respiration of animals.

In Traube's remarkable series of vivisections (Gesammelte Beiträge zaı Pathologie und Physiologie), there are, indeed, indications that, in the difficult breathing produced in rabbits by section of the inferior laryngeal nerve, the lower ribs are drawn inwards, probably by the action of the diaphragm; and the same influence is to be remarked in the cases given by Mr. Le Gros Clark in the Procedings of the Royal Society', vol. xx, p. 125 . 